\title{
Predicting the difficulty in performing a neuraxial blockade
}

\author{
Jong Hae Kim, Seok Young Song, and Baek Jin Kim \\ Department of Anesthesiology and Pain Medicine, School of Medicine, Catholic University of Daegu, Daegu, Korea
}

Background: Traumatic placement of a needle during a neuraxial blockade has been related to many complications such as postdural puncture headache, trauma to neural structures and even spinal hematoma, causing permanent neurologic deficits. Although efforts to minimize the complications caused by traumatic neuraxial blockade have been made, nothing was found to be clear. The authors investigated the predictors of difficult neuraxial blockade using the first puncture success and number of attempts as measures to assess the difficulty.

Methods: In this prospective observational study, 253 patients scheduled for elective surgery underwent spinal or epidural anesthesia. Patient data (age, sex, height, weight, body mass index, and quality of anatomical landmarks), the provider's level of experience, type of blockade (spinal or epidural), needle type/gauge and the distance from skin to subarachnoid or epidural space were recorded. Significant variables were first determined by Student's t-test and Pearson's chi square test and then logistic and Poisson regression tested the association of the first puncture success and number of attempts with the significant variables.

Results: The provider's level of experience and the distance from skin to subarachnoid or epidural space were significant in logistic and Poisson regression. Body mass index was significant only in Poisson regression and the quality of anatomical landmarks was significant only in logistic regression.

Conclusions: Provider's level of experience and the distance from skin to subarachnoid or epidural space influenced the difficulty in performing a neuraxial blockade. (Korean J Anesthesiol 2011; 61: 377-381)

Key Words: Epidural anesthesia, Epidural space, Intraoperative complications, Spinal anesthesia, Subarachnoid space.

Received: April 5, 2011. Revised: May 24, 2011. Accepted: May 26, 2011.

Corresponding author: Jong Hae Kim, M.D., Department of Anesthesiology and Pain Medicine, School of Medicine, Catholic University of Daegu, 3056-6, Daemyeong 4-dong, Nam-gu, Daegu 705-718, Korea. Tel: 82-53-650-4979, Fax: 82-53-650-4517, E-mail: usmed@cu.ac.kr (c) This is an open-access article distributed under the terms of the Creative Commons Attribution Non-Commercial License (http:// creativecommons.org/licenses/by-nc/3.0/), which permits unrestricted non-commercial use, distribution, and reproduction in any medium, provided the original work is properly cited. 


\section{Introduction}

For decades, many complications related to traumatic needle placement during a central neuraxial blockade have been reported. The extent of the complications vary, ranging from self-resolving events, such as postdural puncture headache [1], to severe incidents, such as permanent neurologic sequelae [2]. Some efforts have been made in order to predict the difficult neuraxial blockade for the purpose of minimizing the risk caused by traumatic placement of the needle $[3,4]$. However, no absolute predicting factors have been found so far in spite of the efforts. To determine such factors, we investigated the association of the first puncture success (a successful block achieved by only one skin puncture with no redirection of a needle) and number of attempts (number of skin punctures and redirections of a needle) with factors dependent on the patient, the provider and the technique.

\section{Materials and Methods}

After obtaining approval from the institutional review board and informed consent, this prospective observational study was carried out on 253 consecutive patients undergoing elective surgery requiring neuraxial blockade. All spinals and epidurals were used as the primary anesthetic. The type of block, the level, the approach, the needle and the local anesthetic were based on the preference of the attending anesthesiologist. Identification of the subarachnoid space and the epidural space required the free flow of cerebrospinal fluid and the loss of resistance to saline, respectively. Prior to administration of the epidural or spinal anesthesia, sex, age, height, and weight were recorded from the medical records. Body mass index was calculated as weight divided by the square of height $\left(\mathrm{kg} / \mathrm{m}^{2}\right)$. The quality of anatomical landmarks, categorized as good (well-palpable interspinous space), poor (poorly-palpable interspinous space) or none (not palpable interspinous space) was assessed by physical examination of the patient's lumbar spine. Also, the provider's level of experience was categorized as second-, third-, or fourth-year resident of anesthesiology, staff anesthesiologist, taking-over of an attending staff (an attending staff takes over a difficult procedure which cannot be completed by a resident) and taking-over of more than 2 attending staffs. All trainees were supervised by the attending anesthesiologist. By excluding the first year-residents from this study, all the providers participating in this study have at least 6 months of experience performing a neuraxial blockade. During the procedure, the distance from the skin to the end of the needle was measured with a sterile ruler and this value was subtracted from the total needle length (needle tip to back of hub). The remainder was noted as the distance from skin to subarachnoid or epidural space. Needle type/gauge, the type of the block, the number of attempts (defined as the number of skin punctures and redirections of a needle) and the success or failure of the blockade were recorded after the placement of the block. Successful surgical anesthesia achieved with only one skin puncture and no redirection of a needle was regarded as first puncture success. Otherwise, it was regarded as first puncture failure. Complications occurring during the placement of the block were also recorded. Among the recorded variables, the number of attempts and first puncture success were used to assess difficult neuraxial block.

Statistical analyses were performed using SPSS software (version 14.0, SPSS Inc, IL, USA). The overall success rate and the first puncture success rate were calculated independently. The difference of numerical variables including age, height, weight, body mass index, number of attempts and the distance from skin to subarachnoid or epidural space between first puncture success group and first puncture failure group was analyzed using Student's t-test. Comparison of categorical variables, such as sex, quality of anatomical landmarks, provider's level of experience, needle type/gauge and the type of the block was made by Pearson's chi-square test. Significantly differing variables in univariate analysis were subjected to Logistic regression and Poisson regression, which allows for overdispersion, to test their association with first puncture success and the number of attempts, respectively. A significance level was set at $\mathrm{P}<0.05$.

\section{Results}

The study was conducted in 253 patients consisting of 166 males (65.6\%) and 87 females (34.4\%). The ages ranged from 15 to 93 years. Subarachnoid block and epidural block were performed in 131 (51.8\%) and 122 (48.2\%) patients, respectively. Successful surgical anesthesia was attained in 242 patients (95.7\%) out of 253 patients. First puncture success rate was $44.7 \%$ (113 patients out of 253 patients). No severe events were reported in any of the patients.

Table 1 shows the results of the univariate analysis comparing first puncture success and first puncture failure. There were significant differences between the two groups with respect to body mass index, quality of anatomical landmarks, provider's level of experience, distance from skin to subarachnoid or epidural space and number of attempts. Table 2, 3 and 4 show the relationship of these 4 variables to first puncture success and number of attempts. Staff anesthesiologists had the least risk with regard to number of attempts than any other category (Table 2), while significant differences in first puncture success was found only between staff anesthesiologists and $2^{\text {nd }}$ year residents (Table 3 ). In seven procedures that were completed 
Table 1. Comparison of Patients' and Technical Factors between First Puncture Failure and First Puncture Success Groups

\begin{tabular}{lccc}
\hline & $\begin{array}{c}\text { First puncture failure } \\
(\mathrm{n}=140)\end{array}$ & $\begin{array}{c}\text { First puncture success } \\
(\mathrm{n}=113)\end{array}$ & P \\
\hline Age (yr) & $56.2 \pm 1.43$ & $56.0 \pm 1.51$ & 0.907 \\
Sex $(\mathrm{M} / \mathrm{F})$ & $92 / 48$ & $74 / 39$ & 0.970 \\
Height $(\mathrm{cm})$ & $163.2 \pm 0.69$ & $163.7 \pm 0.84$ & 0.657 \\
Weight $(\mathrm{kg})$ & $63.6 \pm 0.90$ & $61.5 \pm 1.06$ & 0.138 \\
Body mass index $\left(\mathrm{kg} / \mathrm{m}^{2}\right.$ ) & $23.8 \pm 0.29$ & $22.9 \pm 0.31$ & $0.041^{\dagger}$ \\
Landmarks (good/poor or none) & $86 / 54$ & $95 / 18$ & $0.000^{\ddagger}$ \\
Provider (1-6)* & $36 / 13 / 57 / 25 / 7 / 2$ & $5 / 8 / 78 / 22 / 0 / 0$ & $0.000^{\ddagger}$ \\
Spinal/epidural & $74 / 66$ & $57 / 56$ & 0.702 \\
Needle type $(25 \mathrm{G} / 23 \mathrm{G} / 17 \mathrm{G})$ & $13 / 61 / 66$ & $10 / 47 / 56$ & 0.930 \\
Distance $(\mathrm{cm})$ & $5.2 \pm 0.11$ & $4.9 \pm 0.08$ & $0.016^{\dagger}$ \\
Number of attempts & $5.5 \pm 0.56$ & 1 & $0.000^{\dagger}$ \\
\hline
\end{tabular}

Values are mean \pm SEM or number of patients. Landmarks: quality of anatomical landmarks, Distance: distance from skin to subarachnoid or epidural space. $* 1: 2^{\text {nd }}$ year resident, $2: 3^{\text {rd }}$ year resident, 3: $4^{\text {th }}$ year resident, 4: Staff anesthesiologist, 5: Taking over of an attending staff, 6: Taking over of more than 2 attending staffs. ${ }^{\dagger} \mathrm{P}<0.05$ based on student's t-test, ${ }^{\dagger} \mathrm{P}<0.05$ based on Pearson's chi-square test.

Table 2. Relative Risk and Number of Attempts According to Level of Experience

\begin{tabular}{lccc}
\hline & Relative risk (95\% CI) & Number of attempts & P \\
\hline Attending staff (47, reference group) & 1 & $3.3 \pm 0.46$ & $3.7 \pm 0.63$ \\
$2^{\text {nd }}$ year resident (41) & $2.42(1.84-3.18)$ & $4.7 \pm 1.62$ & $0.000^{*}$ \\
$3^{\text {rd }}$ year resident (21) & $2.00(1.41-2.85)$ & $3.0 \pm 0.50$ & $0.000^{*}$ \\
$4^{\text {th }}$ year resident (135) & $1.67(1.32-2.10)$ & $7.9 \pm 2.19$ & $0.000^{*}$ \\
Taking over of an attending staff (7) & $2.21(1.52-3.23)$ & $17.5 \pm 0.50$ & $0.000^{*}$ \\
Taking over of more than 2 attending staffs (2) & $3.48(2.22-5.45)$ & $0.000^{*}$ \\
\hline
\end{tabular}

Values are number of patients, relative risk (95\% confidence interval) or mean \pm SEM. $* \mathrm{P}<0.05$ based on Poisson regression analysis.

Table 3. Odds Ratio According to Anatomical Landmarks, Distance from Skin to Subarachnoid or Epidural Space and Level of Experience

\begin{tabular}{lccc}
\hline & Odds ratio $(95 \% \mathrm{CI})$ & First puncture success $(\%)$ & $\mathrm{P}$ \\
\hline Landmarks (good/poor or none) & $0.37(0.18-0.74)$ & $53.7 / 25.7$ & $0.005^{*}$ \\
Staff $(47$, reference group) & 1 & 46.8 & $0.000^{*}$ \\
$2^{\text {nd }}$ year resident $(41)$ & $0.10(0.03-0.32)$ & 12.2 & \\
\hline
\end{tabular}

Values are number of patients or odds ratio (95\% confidence interval). Landmarks: quality of anatomical landmarks. ${ }^{*} \mathrm{P}<0.05$ based on logistic regression analysis.

Table 4. Increase Rate of Risk or Odds Per One Unit Increase According to Body Mass Index and Distance from Skin to Subarachnoid or Epidural Space

\begin{tabular}{lcc}
\hline & Increase rate $(95 \% \mathrm{CI})$ & $\mathrm{P}$ \\
\hline Body mass index $\left(\right.$ risk $\left./ \mathrm{kg} / \mathrm{m}^{2}\right)$ & $1.15(1.13-1.17)$ & $0.000^{*}$ \\
Distance (risk/cm) & $1.43(1.33-1.53)$ & $0.000^{*}$ \\
Distance (odds $/ \mathrm{cm})$ & $0.70(0.50-0.95)$ & $0.024^{\dagger}$ \\
\hline
\end{tabular}

Values are increase rate of risk or odds ( $95 \%$ confidence interval). Distance: distance from skin to subarachnoid or epidural space. ${ }^{*} \mathrm{P}<0.05$ based on Poisson regression analysis, ${ }^{\dagger} \mathrm{P}<0.05$ based on logistic regression.

by a staff member after the first provider experienced difficulty, the staff member was successful in reaching the subarachnoid space or epidural space with less than three attempts. However, in two procedures in which more than two staff members participated in completing a neuraxial blockade after the first provider experienced difficulty, the number of attempts for successful surgical anesthesia was more than 15 . The distance from skin to subarachnoid or epidural space was significantly associated with the number of attempts and first puncture success (Table 4). The quality of anatomical landmarks and body mass index were found to be significant regarding first puncture success and number of attempts, respectively (Table 3 and 4).

Unanticipated dural puncture occurred during an epidural block in one patient. Three patients including this patient developed postdural puncture headache. Among them, one patient presented with severe back pain. Bloody tap was noted during the spinal block in two patients but, another skin puncture resulted in a successful free flow of cerebrospinal fluid. 


\section{Discussion}

This study demonstrated that the distance from skin to subarachnoid space or epidural space and the provider's level of experience are associated with difficulty in performing a neuraxial blockade. We used first puncture success (described as a successful surgical anesthesia with only one skin puncture without redirecting the needle) and the number of attempts including new skin punctures and redirections of a needle, as measures to assess the predicting factors for difficult neuraxial block. These were chosen based on the fact that minimizing the number of new skin punctures and redirections of a needle when placing a blockade reduces the patient's discomfort, the risk of complications and assures agility to the surgical schedule. Because it was a very stringent criteria, the first puncture success rate was very low (44.7\%) and factors contributing to the prediction of difficulty in the placement of the block were different from the results of previous studies, likely due to different study designs. The quality of the patients' anatomical landmarks, the adequacy of patient positioning and the provider's level of experience were important predictors of successful location of the subarachnoid or epidural space at the first skin puncture without needle redirections [3]. When successful needle placement at the initial spinal level and the number of new skin punctures were used to assess the difficulty encountered in performing the block, the quality of landmarks and obvious anatomical deformities predicted difficulty of a neuraxial block [4].

Methodological diversity of categorizing the provider's level of experience, various measures to assess the difficulty of neuraxial blocks and interindividual variability in manual dexterity likely contributes to the conflicting results in each study, including the present study. For example, in our study, staff anesthesiologists with over 6 years of clinical experience had a greater first puncture success rate than $2^{\text {nd }}$ year residents with at least 6 months of clinical experience (Table 3). The staff anesthesiologists also had the least risk in finding the subarachnoid or epidural space among the medical providers (Table 2). The agreement of this result can be found in other studies in which the training level was significantly associated with difficulty of neuraxial blocks $[3,5,6]$. In particular, exclusion of residents having clinical experience less than 6 months in the current study had an association with difficulty in neuraxial blocks. This result differs from the previous report which explained that the inclusion of first year residents with experience less than 6 months of training might have accounted for the discrepancy between studies [3]. In addition, the result in this study disagrees with those which could not find such an association $[4,7]$. Furthermore, there exists interindividual variability in success of identifying the epidural space at the first attempt at one intervertebral space [8] and the number of spinal needle insertions to reach the subarachnoid space, even among fully trained staff members [9]. In our study, two procedures which required over 15 attempts by more than two staff anesthesiologists, reflects the absolute difficulty and the above mentioned interindividual variability.

The fact that measurement of the distance from skin to either the subarachnoid or epidural space in the current study is not possible unless the subarachnoid or epidural space is actually found during a procedure may limit the value of the distance for predicting the difficulty of a neuraxial blockade. However, the preoperative evaluation of the patients' lumbar spines using noninvasive diagnostic imaging such as plain radiography, computed tomography [10], magnetic resonance imaging [11] and ultrasonography $[12,13]$ helps the estimation of the distance [12], although the correlation of the measured value during the block with the value derived from the above preoperative imaging has yet to be studied due to discrepancy of the values caused by differences in the patient's posture and the needle trajectory between each condition of measurements.

In principle, a midline approach was chosen first for placement of the blockade in this study. Nevertheless, another approach was allowed when difficulty was met during the procedure because it is impractical to be insistent on the first chosen approach in spite of the difficulty in placing the block. In addition, the likelihood that the essentially blind nature of this approach to the epidural or subarachnoid space would result in a true midline entry of the space relatively infrequently makes the differentiation of a midline approach from a paramedian approach meaningless. Even if the procedure was successful with a blind midline approach, there exists the probability that it was actually accomplished with a paramedian or parasagittal approach.

In this study, body mass index was significantly associated with difficulty only with regard to the number of attempts. However, our findings support the interpretation that it is a minor predictor of difficulty, since it can be thought that body mass index is one of the factors which influence the distance from skin to subarachnoid or epidural space. This is supported by previous studies that have demonstrated correlations between the depth of the epidural space and body mass index [14-18]. In addition, Sprung et al. also reported body mass index was very weakly related to the difficulty of a neuraxial block [4].

In previous studies, the quality of anatomical landmarks was strongly associated with successful anesthesia at the first attempt [3] and it was an independent predictor of successful spinal or epidural needle placement at the first chosen interspace and the number of skin punctures [4]. In this study, the quality of anatomical landmarks differed between first puncture success and first puncture failure groups in univariate 
analysis and it was associated with first puncture success. These findings were similar with the former study. However, like the above mentioned body mass index, we regarded the quality of anatomical landmarks as a minor predictor of difficulty, since it was significantly associated only in one of the two regressions used in this study. In actuality, the distance from skin to subarachnoid or epidural space, body mass index and the quality of anatomical landmarks are closely related to each other. In fact, it can be assumed that combination of the two minor predictors made the distance a major predictor in this study.

We discarded variables which were not different between first puncture success group and first puncture failure group at the univariate analysis during the logistic and Poisson regressions because of both their insignificant $\mathrm{P}$ and inability to increase the predictive capability of the model. Therefore, the possibility that there are other factors influencing the difficulty of a neuaxial blockade, which have not been included in this study, cannot be excluded.

Our results suggest that greater chance of first puncture success followed by minimized complications would be more likely in patients having a closer distance from skin to subarachnoid or epidural space. Furthermore, more experienced anesthesiologists are likely to have a better chance of reaching the subarachnoid or epidural space at the first skin puncture without redirection of a needle. As the distance from skin to subarachnoid or epidural space cannot be altered, special attention should be given to the provider's level of experience in the management of difficult neuraxial blockade.

\section{Acknowledgements}

This work was supported by research grants from the Catholic University of Daegu in 2010.

\section{References}

1. Gaiser R. Postdural puncture headache. Curr Opin Anaesthesiol 2006; 19: 249-53.

2. Auroy Y, Narchi P, Messiah A, Litt L, Rouvier B, Samii K. Serious complications related to regional anesthesia: Results of a prospective survey in france. Anesthesiology 1997; 87: 479-86.

3. de Filho GR, Gomes HP, da Fonseca MH, Hoffman JC, Pederneiras SG, Garcia JH. Predictors of successful neuraxial block: A prospec- tive study. Eur J Anaesthesiol 2002; 19: 447-51.

4. Sprung J, Bourke DL, Grass J, Hammel J, Mascha E, Thomas P, et al. Predicting the difficult neuraxial block: A prospective study. Anesth Analg 1999; 89: 384-9.

5. Kestin IG. A statistical approach to measuring the competence of anaesthetic trainees at practical procedures. Br J Anaesth 1995; 75: 805-9.

6. Kopacz DJ, Neal JM, Pollock JE. The regional anesthesia "learning curve". what is the minimum number of epidural and spinal blocks to reach consistency? Reg Anesth 1996; 21: 182-90.

7. Munhall RJ, Sukhani R, Winnie AP. Incidence and etiology of failed spinal anesthetics in a university hospital: A prospective study. Anesth Analg 1988; 67: 843-8.

8. Dashfield AK, Coghill JC, Langton JA. Correlating obstetric epidural anaesthesia performance and psychomotor aptitude. Anaesthesia 2000; 55: 744-9.

9. Tessler MJ, Kardash K, Wahba RM, Kleiman SJ, Trihas ST, Rossignol M. The performance of spinal anesthesia is marginally more difficult in the elderly. Reg Anesth Pain Med 1999; 24: 126-30.

10. Harrison GR. Topographical anatomy of the lumbar epidural region: An in vivo study using computerized axial tomography. $\mathrm{Br} \mathrm{J}$ Anaesth 1999; 83: 229-34.

11. Capogna G, Celleno D, Simonetti C, Lupoi D. Anatomy of the lumbar epidural region using magnetic resonance imaging: $\mathrm{A}$ study of dimensions and a comparison of two postures. Int J Obstet Anesth 1997; 6: 97-100.

12. Grau T, Leipold RW, Conradi R, Martin E, Motsch J. Ultrasound imaging facilitates localization of the epidural space during combined spinal and epidural anesthesia. Reg Anesth Pain Med 2001; 26: 64-7.

13. Grau T, Leipold RW, Horter J, Conradi R, Martin E, Motsch J. The lumbar epidural space in pregnancy: Visualization by ultrasonography. Br J Anaesth 2001; 86: 798-804.

14. Hoffmann VL, Vercauteren MP, Buczkowski PW, Vanspringel GL. A new combined spinal-epidural apparatus: Measurement of the distance to the epidural and subarachnoid spaces. Anaesthesia 1997; 52: 350-5.

15. Meiklejohn BH. Distance from skin to the lumbar epidural space in an obstetric population. Reg Anesth 1990; 15: 134-6.

16. Watts RW. The influence of obesity on the relationship between body mass index and the distance to the epidural space from the skin. Anaesth Intensive Care 1993; 21: 309-10.

17. Harrison GR, Clowes NW. The depth of the lumbar epidural space from the skin. Anaesthesia 1985; 40: 685-7.

18. Stamatakis E, Moka E, Siafaka I, Argyra E, Vadalouca A. Prediction of the distance from the skin to the lumbar epidural space in the greek population, using mathematical models. Pain Pract 2005; 5: $125-34$. 